\title{
Thermonuclear Power Plants
}

\author{
HSUE-SHEN TSIEN 1
}

Daniel and Florence Guggenheim Jet Propulsion Center, California Institute of Technology, Pasadena, Calif.

\begin{abstract}
Some of the unique features of thermonuclear power plants and the essential problems in the technical design of such plants are discussed in this paper. The thermonuclear reaction rate for the fusion of deuterium is calculated on the basis of a similar analysis published by Gamow and Teller. The pressure, temperature, and minimum dimensions of the necessary reaction chamber are determined largely by consideration of reaction quenching and energy loss near the walls. Results are presented for the power output and the efficiency of a power station utilizing the deuterium fusion reaction. The comment by Greenstein that follows this paper deals particularly with the difficult problem of calculating the reaction quenching and energy loss rates at the walls.
\end{abstract}

\section{Introduction}

A GREAT majority of the present discussions and plans on the utilization of nuclear energy for power production is related to the fission reaction. Although the nuclear fission power plants have many distinct advantages over the conventional power plants, the limited world reserve of uranium and thorium that can be practically mined makes the long term prospect of such power plants somewhat uncertain. On the other hand, the thermonuclear fusion reaction, particularly the "burning" of deuterium into helium, utilizes a very abundant fuel. Therefore, if the fusion reaction can be made to generate energy for power plants, the prospect of world energy supply will be very much brighter.

But can the fusion reaction be utilized in terrestrial power plants? This question has been examined by E. Sänger and his collaborators $(1,2) .^{2}$ However, a critical reading of Sänger's work will show that part of his analysis is not valid because he has not gone deep enough into the subject. It is the purpose of this note to point out some of the unique features of the thermonuclear power plants and the essential problems in the technical design of such power plants. It will be seen that such engineering projects are truly of stupendous proportions and are a challenge to one's imagination. However, the reward to the welfare of the human race by a successful development of thermonuclear power plants is so great as to make the careful examination of this problem a very worthwhile research project.

\section{Thermonuclear Reaction Rate}

Thermonuclear reactions are reactions between charged nuclei. Because of the electric charge, necessarily positive, the approach of the nuclei to each other has to overcome the Coulomb repulsion between the nuclei. Therefore, only if the relative kinetic energy of the nuclei is high, can a close enough approach be obtained and a reaction take place. This required kinetic energy is so large that even at temperatures as high as $10^{8} \mathrm{~K}$ only nuclei in the high energy end of the Maxwellian velocity distribution can achieve reaction. Therefore only a very small fraction of the nuclei participate in the reaction. In other words, the reaction rate is quite small. This observation leads to a great simplification of the calcula-

Received Oct. 24, 1955.

1 Formerly: Robert H. Goddard Professor of Jet Propulsion.

${ }^{2}$ Numbers in parentheses indicate References at end of paper. tion: The nuclear distribution can be considered as quasisteady; i.e., the Maxwellian distribution can in fact be used in spite of the slight lack of thermodynamic equilibrium during the reaction. Gamow and Teller (3) have developed such a theory of thermonuclear reactions. The following is a slightly modified form of the theory suitable for the present purpose.

Considering the colliding particles as rigid spheres, it is well known (4) that the number $d N$ of collisions per unit volume per unit time between particles of type 1 and 2 with kinetic energy of collision between $\epsilon$ and $\epsilon+d \epsilon$ is

$$
d N=\frac{n_{1} n_{2}}{s}\left(\frac{2 \pi k T}{\mu}\right)^{1 / 2} e^{-\epsilon / k T_{2} D_{12}{ }^{2}} \frac{\epsilon}{k T} \frac{d \epsilon}{k T} \ldots \ldots
$$

where $n_{1}, n_{2}$ are the number densities of particles of type 1 and 2 , respectively; $s$ is the symmetry number, equal to 2 if type 1 and type 2 are the same, and equal to 1 if not; $\mu$ is the reduced mass of type 1 and type 2 particles. $D_{12}$ is the average diameter of the two types of particles; i.e., if $D_{1}$ and $D_{2}$ are the diameters of type 1 and 2 particles, then

$$
D_{12}=\frac{1}{2}\left(D_{1}+D_{2}\right) \ldots
$$

To put this general formula into a form more useful for the present computation, molar fractions $\nu_{1}$ and $\nu_{2}$ are introduced

$$
\nu_{1}=\frac{n_{1}}{n} \quad \nu_{2}=\frac{n_{2}}{n} \quad n=\Sigma_{n i} \ldots \ldots \ldots
$$

where $n$ is the total number of particles per unit volume; the particles may include electrons besides the nuclei. Furthermore, if $M_{1}$ and $M_{2}$ are the mass of particles of type 1 and 2, and $A_{1}$ and $A_{2}$ are the corresponding quantities expressed in terms of atomic mass units ("atomic weights" of the nuclei), and $M$ is the mass of one atomic mass unit, then

$$
\mu=\frac{M_{1} M_{2}}{M_{1}+M_{2}}=\frac{A_{1} A_{2}}{A_{1}+A_{2}} M=A M .
$$

Thus $A$ is the reduced mass expressed in terms of atomic mass units. If $V$ is the relative velocity of the two colliding particles, then $\epsilon$, the relative translational energy, is defined as

$$
\epsilon=\frac{1}{2} \mu V^{2} \ldots \ldots \ldots \ldots \ldots \ldots \ldots
$$

If $P$ is the thermodynamic pressure, then

$$
n=P / k T \ldots
$$

Equation [6] is true only if the assembly of particles is at thermodynamic equilibrium and if the particles essentially do not interact, i.e., the assembly is a perfect gas. At the extremely low gas density that will be considered, this is true to a high degree of accuracy. Of course, if the sum of the particles is not at thermodynamic equilibrium, e.g., fusion product neutrons which hardly collide a sufficient number of times with other particles to have a Maxwellian distribution of velocity within the dimension of region considered, then such particles must not be considered in calculating the "total" particle density $n$. The "pressure" produced by such particles on the wall of the containing vessel has to be treated separately. On the other hand, photons, if any, that are almost at thermodynamic equilibrium must be included in the particle density $n$. 
The most important modification of [1] is the following: Nuclear reactions cannot be considered as collisions between rigid spheres but are expressed through an effective cross section $\sigma$. The effective cross section for collision of rigid spheres is a circle of radius $D_{12}$. Therefore

$$
D_{12}^{2}=\sigma / \pi
$$

By substituting [3], [4], [6], and [7] into [1], the number $d N$ is

$$
d N=\frac{4 \nu_{1} \nu_{2} \sigma P^{2}}{s \sqrt{2 \pi A M}} \frac{e^{-\epsilon / k T}}{(k T)^{7 / 2}} \epsilon d \epsilon \ldots \ldots \ldots \ldots
$$

It is important to note that in general the effective cross section is not a constant but function of energy $\epsilon$. In particular, according to the theory of nuclear reaction, the quantal penetration of Coulomb barrier gives the effective cross section $\sigma$ as

$$
\begin{aligned}
\sigma=\frac{\Lambda^{2}}{4 \pi} \frac{\Gamma A M R^{2}}{\not h^{2}} \times \\
\quad \exp \left[-\frac{2 \pi e^{2} Z_{1} Z_{2} \sqrt{A M}}{\not h \sqrt{2 \epsilon}}+\frac{4 e \sqrt{2 A M Z_{1} Z_{2} R}}{\not h}\right] \ldots
\end{aligned}
$$

where $\Lambda$ is the de Broglie wavelength

$$
\Lambda=\frac{2 \pi \not h}{\sqrt{2 A M \epsilon}} .
$$

$R$ is the radius of the compound nucleus formed during reaction, and can be estimated as

$$
R=\left[1.7+1.22\left(A_{1}+A_{2}\right)^{1 / 3}\right] \times 10^{-13} \mathrm{~cm} .
$$

$\Gamma$ is the half-width of the nuclear resonance level. $Z_{1}$ and $Z$ are the nuclear charges in units of electronic charge $e$.

By substituting [9] and [10] into [8], it is seen that the variation of $d N / d \epsilon$ with respect to $\epsilon$ is due to the exponential factor

$$
\exp \left\{-\left[\frac{\epsilon}{k T}+\frac{2 \pi e^{2} Z_{1} Z_{2} \sqrt{A M}}{\not h \sqrt{ } 2 \epsilon}\right]\right\} .
$$

There is a minimum of the quantity within the square bracket with respect to $\epsilon$ which corresponds to the maximum of $d N / d \epsilon$. If this $\epsilon$ is denoted by $\epsilon^{*}$, then

$$
\frac{1}{k T}=\frac{\pi e^{2} Z_{1} Z_{2} \sqrt{A M}}{\sqrt{2} \not h \epsilon^{* 3 / 2}}
$$

or

$$
\epsilon^{*}=\left\{\frac{\pi e^{2} Z_{1} Z_{2} \sqrt{A M} k T}{\sqrt{2} \not / 4}\right\}^{2 / \%} \ldots
$$

Near this value of $\epsilon$, the expression in the square bracket of [12] can be approximated by

$$
\begin{gathered}
\frac{\epsilon}{k T}+\frac{2 \pi e^{2} Z_{1} Z_{2} \sqrt{A M}}{\not h \sqrt{2 \epsilon}} \cong \frac{\epsilon^{*}}{k T}+\frac{2 \pi e^{2} Z_{1} Z_{2} \sqrt{A M}}{\sqrt{2} \not h \sqrt{\epsilon^{*}}}+\frac{1}{2} \cdot \frac{3}{2} \times \\
\frac{\pi e^{2} Z_{1} Z_{2} \sqrt{A M}}{\sqrt{2} \not h \epsilon^{* / 2}}\left(\epsilon-\epsilon^{*}\right)^{2}=3 \frac{\epsilon^{*}}{k T}+\frac{3}{4} \frac{1}{k T \epsilon^{*}}\left(\epsilon-\epsilon^{*}\right)^{2} .
\end{gathered}
$$

Thus, under this approximation, $d N$ can be computed as

$$
\begin{array}{r}
d N=\frac{4 \nu_{1} \nu_{2} \Gamma P^{2}}{s \sqrt{2 \pi A M(k T)^{7 / 2}} \frac{\pi R^{2}}{2} \exp }\left[\frac{4 e \sqrt{2 A M Z_{1} Z_{2} R}}{\not h}-\frac{3 \epsilon^{*}}{k T}\right] \times \\
\exp \left[-\frac{3}{4} \frac{1}{k T \epsilon^{*}}\left(\epsilon-\epsilon^{*}\right)^{2}\right] d \epsilon \ldots[15]
\end{array}
$$

By integrating over all $\epsilon$, we have the number $N$ of effective binary collisions per unit vol per unit time as

$$
\begin{array}{r}
N=\frac{4 \pi \nu_{1} \nu_{2} R^{2}}{s \sqrt{3}}\left(\frac{P}{k T}\right)^{2}\left(\frac{\mathrm{r}}{k T}\right) \sqrt{\frac{k T}{2 A M} \sqrt{\frac{\epsilon^{*}}{k T}} \times} \\
\quad \exp \left[\frac{4 e \sqrt{2 A M Z_{1} Z_{2} R}}{\not h}-3 \frac{\epsilon^{*}}{k T}\right]
\end{array}
$$

From [13]

$$
\frac{\epsilon^{*}}{k T}=\left(\frac{\pi^{2} e^{4} Z_{1}^{2} Z_{2}^{2} A M}{2 \not L^{2} k T}\right)^{1 / 3} .
$$

Equations [16] and [17] together determine the reaction rate. There is only one important difference between [16] and the original formula due to Gamow and Teller (3); Gamow and Teller have not included the symmetry number $s$ and thus may be wrong in some cases by a factor of 2 .

If we denote by $x$ the quantity $(k T)^{1 / 3}$, then the temperature dependent part of $[16]$ can be written as

$$
x^{8} \exp \left[-3\left(\frac{\pi^{2} e^{4} Z_{1}^{2} Z_{2}^{2}}{2 \not h^{2}}\right)^{1 / s} x\right] .
$$

This quantity clearly has a maximum at some value of $x$, say $x_{0} ; x_{0}$ is determined by

$$
8-3\left(\frac{\pi^{2} e^{4} A M Z_{1}{ }^{2} Z_{2}^{2}}{2 \not h^{2}}\right)^{1 / 3} x_{0}=0
$$

Equation [19] gives the optimum reaction temperature $T_{0}$ for maximum reaction rate at constant pressure as

$$
T_{0}=\left(\frac{3}{8}\right)^{3} \frac{\pi^{2} e^{4} A Z_{1}^{2} Z_{2}^{2}}{2 k h^{2}}
$$

By putting in the numerical values of physical constants

$$
T_{0}=1.442 \times 10^{8} A Z_{1}{ }^{2} Z_{2}^{2}{ }^{\circ} \mathrm{K} \ldots \ldots \ldots \ldots
$$

Equations [20] and [21] show that the optimum reaction temperature depends only on the reduced mass and charges of the nuclei and is independent of the details of the reaction. $T_{0}$ is the smallest for proton-proton reaction, $(A=1 / 2$, $Z_{1}=Z_{2}=1$ ), for which $T_{0}=0.721 \times 10^{8} \mathrm{~K}$.

The important parameter in the expression of reaction rate is the level width $\Gamma$. This has to be determined experimentally. However the experimental reaction cross sections are usually expressed as

$$
\sigma=\frac{B}{\epsilon} e^{-C / \sqrt{\epsilon}}
$$

where $B$ and $C$ are two empirically determined constants for any one reaction. Same as the preceding paragraphs, [8] and [22] can be combined to give the formulas

$$
\begin{array}{r}
\frac{\epsilon^{*}}{k T}=\left(\frac{C^{2}}{4 k T}\right)^{1 / 3} \ldots \ldots \ldots \ldots \ldots \ldots \\
N=\frac{4 \nu_{1} \nu_{2}}{s}\left(\frac{B}{k T}\right)\left(\frac{P}{k T}\right)^{2} \sqrt{\frac{2 k T}{3 A M}} \sqrt{\frac{\epsilon^{*}}{k T}} e^{-3\left(\epsilon^{*} / k T\right)} \ldots
\end{array}
$$

and

$$
T_{0}=\left(\frac{3}{8}\right)^{3} \frac{C^{2}}{4 k}
$$

If the optimum temperature $T_{0}$ is used as a reference temperature

$$
\frac{\epsilon^{*}}{k T}=\frac{8}{3}\left(\frac{T_{0}}{T}\right)^{1 / 2}
$$


For numerical computation, $P$ is usually given in atmospheres. Thus

$$
\frac{P}{k T}=7.34 \times 10^{21} \frac{P}{T} \quad \mathrm{~cm}^{-3} \ldots \ldots \ldots \ldots
$$

$B$ is usually given in units of barns-kilovolts, thus

$$
\frac{B}{k T}=1.160 \times 10^{-17} \frac{B}{T} \quad \mathrm{~cm}^{2} \ldots \ldots \ldots \ldots
$$

$C$ is usually given in units of $\mathrm{kv}^{1 / 2}$, thus

$$
T_{\theta}=1.528 \times 10^{5} C^{2}
$$

If $E$ is the energy production of a single binary reaction, then the rate of energy production $Q$ per unit vol per unit time is obviously

$$
Q=E N
$$

\section{Example: Deuterium Reaction}

Because of the abundance of deuterium as a naturally occurring stable isotope of hydrogen, it is of interest to consider the burning of deuterium. The accurate reaction data were given recently by Arnold et al. (5)

$$
\sigma=\frac{B^{\prime}}{\epsilon^{\prime}} e^{-C^{\prime} / \sqrt{\epsilon^{\prime}}}
$$

where $\epsilon^{\prime}$ is the deuteron energy in kilovolts in the usual laboratory coordinate system, and $B^{\prime}=288$ barns-kv, $C^{\prime}=45.7$ $\mathrm{kv}{ }^{1 / 2}$. Since the $\epsilon$ in $[22]$ is the relative kinetic energy defined by (5) and the ratio of deuterium mass and the reduced mass is 2 in a deuteron-deuteron reaction,

$$
\epsilon^{\prime}=2 \epsilon
$$

Therefore, by using Arnold's data, the reaction constants $B$ and $C$ are

$$
\begin{aligned}
B & =B^{\prime} / 2=144 \text { barnes-kv } \ldots \ldots \ldots \ldots \\
C & =C^{\prime} / \sqrt{2}=32.36 \mathrm{kv}^{1 / 2} \ldots \ldots \ldots \ldots
\end{aligned}
$$

Equation [29] immediately gives the optimum temperature $T_{0}$ for deuteron-deuteron reaction as

$$
T_{0}=1.600 \times 10^{8^{\circ}} \mathrm{K}
$$

According to Arnold et al., the deuteron-deuteron reaction branches, with almost equal probability, into two reactions

$$
\begin{aligned}
& { }_{1} \mathrm{H}^{2}+{ }_{1} \mathrm{H}^{2} \rightarrow{ }_{1} \mathrm{H}^{3}+{ }_{1} p^{1} \ldots \ldots \ldots \ldots \ldots[36] \\
& { }_{1} \mathrm{H}^{2}+{ }_{1} \mathrm{H}^{2} \rightarrow{ }_{2} \mathrm{He}^{3}+{ }_{0} n^{1} \ldots \ldots \ldots \ldots \ldots[37]
\end{aligned}
$$

Since the masses of the atomic species are given as follows

$$
\begin{aligned}
& A\left({ }_{1} \mathrm{H}^{2}\right)=2.014735 \\
& A\left({ }_{1} \mathrm{H}^{3}\right)=3.016997 \\
& A\left({ }_{2} \mathrm{He}^{3}\right)=3.016977 \\
& A\left({ }_{1} p^{1}\right)=1.008142 \\
& A\left({ }_{0} n^{1}\right)=1.008982
\end{aligned}
$$

The reaction [36] then produces

$$
\begin{array}{r}
2 \times 2.014735-(3.016997+1.008142)=0.04331 \mathrm{amu}= \\
4.03 \mathrm{Mev}=6.46 \times 10^{-6} \mathrm{ergs} \ldots
\end{array}
$$

The reaction $[37]$ produces

$$
\begin{array}{r}
2 \times 2.014735-(1.008982+3.016177)=0.003511 \mathrm{amu}= \\
3.27 \mathrm{Mev}=5.24 \times 10^{-6} \mathrm{ergs} \ldots \ldots
\end{array}
$$

However, in a thermonuclear reaction chamber, [36] and
[37] do not represent the end of reactions. The reaction products are immediately thermalized by elastic collisions with other particles; then the following reactions are possible

$$
\begin{aligned}
& { }_{2} \mathrm{He}^{3}+{ }_{0} n^{1} \rightarrow{ }_{1} \mathrm{H}^{3}+{ }_{1} p^{1} \ldots \ldots \ldots \ldots \ldots \\
& { }_{1} \mathrm{H}^{3}+{ }_{1} \mathrm{H}^{2} \rightarrow{ }_{2} \mathrm{He}^{4}+{ }_{0} n^{1} \ldots \ldots \ldots \ldots \ldots \ldots \\
& { }_{2} \mathrm{He}^{3}+{ }_{1} \mathrm{H}^{2} \rightarrow{ }_{2} \mathrm{He}^{4}+{ }_{1} p^{1} \ldots \ldots \ldots \ldots \ldots
\end{aligned}
$$

At first sight it seems that the reaction [41] depending upon both components of the reaction products of low concentration will be very much less frequent than [42] and [43] depending upon only one component of reaction product. However at thermal energies corresponding to temperatures of the order of $10^{8} \mathrm{~K}$, the cross section of [41] is of the order of 10 barns, while according to Arnold (5), the reaction cross sections of [42] and [43] are very much smaller. Therefore [41] is in fact the only reaction of importance while [42] and [43] can be neglected. This being the case, then eventually all of the deuteron-deuteron reaction really results in the production of tritium according to [36]. Therefore the average energy production $E$ for every deuteron-deuteron reaction is according to [39]

$$
E=6.46 \times 10^{-6} \mathrm{ergs} .
$$

The energy produced by burning a unit mass of deuterium is thus

$$
\frac{6.46 \times 10^{-6} \times 2.388 \times 10^{-8} \times 0.605 \times 10^{24}}{4.029} \mathrm{cal} / \mathrm{gr}=
$$

Now assume $T=T_{0}=1.600 \times 10^{8} \mathrm{~K}, P=100 \mathrm{~atm}$. The feed gas $D_{2}$ will be completely ionized at this temperature, and reacting mixture will be composed initially of equal numbers of deuterons and electrons. Thus $\nu_{1}=\nu_{2}=1 / 2$, $s=2$. Then using [24], [26], [30] the energy production $Q$ per unit vol per unit time is

$$
\begin{gathered}
Q=6.46 \times 10^{-6} \times \frac{1}{2} \times \frac{1.160 \times 10^{-17} \times 144}{1.600 \times 10^{8}} \times \\
\left(\frac{7.34 \times 10^{23}}{1.600 \times 10^{8}}\right)^{2} \sqrt{\frac{8}{3} \times \frac{2 \times 8.316 \times 10^{7} \times 1.6 \times 10^{8}}{3 \times 1.007}} \times \\
e^{-8} \mathrm{ergs} / \mathrm{cm}^{3} \mathrm{sec}=0.365 \times 10^{8} \mathrm{ergs} / \mathrm{cm}^{3} \mathrm{sec}= \\
3.65 \mathrm{watts} / \mathrm{cm}^{3}=0.874 \mathrm{cal} / \mathrm{cm}^{3} \mathrm{sec} \ldots \ldots
\end{gathered}
$$

It is interesting to note that this volume rate of energy generation is only $1 / 10$ of the rate of generation as in a modern aircraft gas turbine combustion chamber using hydrocarbon fuel. Therefore in spite of the extremely high temperature, thermonuclear reaction using deuterium is a relatively slow reaction. The reason for this anomaly is the extremely low density of the hot gas: There simply are not enough deuterons in a unit volume to give high reaction rate. However, as Sänger (1) has shown, other nuclei generally give even lower rates of energy production.

\section{Thermonuclear Reaction Chamber}

The moderate volume rate of energy production together with the extremely high gas temperature naturally call ones attention to the problem of quenching of the "flame" by excessive cooling. This problem is in fact the central problem of thermonuclear reaction chamber. There is certainly a critical size, say a critical diameter, of the reaction chamber below which the reaction cannot be maintained. As a very rough first estimate, one may take the chemical combustion as a model, and use the mean free path as the sizing length. Because of the relatively slow thermonuclear reaction, the chemical model should be one of poor reactivity. Thus the quenching diameter at atmospheric pressure can be taken as 
$1 \mathrm{~cm}$. The pressure effect on quenching can be thought of as a Reynolds number effect. Then the quenching diameter at $100 \mathrm{~atm}$ will be $1 / 100 \mathrm{~cm}$. To translate this value to thermonuclear reaction chamber, one notes the fact that the ratio of mean free path for the two cases is approximately $10^{6}$. Thus the rough estimate of the critical diameter $D_{c}$ of the reaction chamber is

$$
D_{c}=\frac{1}{100} \times 10^{6} \mathrm{~cm}=100 \text { meters. } \ldots \ldots \ldots
$$

If the length is to be ten times the diameter, then the thermonuclear reaction chamber is a vessel of 100 meters diam and 1000 meters long built to withstand a pressure of 100 atmospheres!

To examine the quenching problem in some detail, one must first estimate the mean free path of the fully ionized mixture of deuteron and electron. If $n$ is the particle density, and if $\sigma_{s}$ is the scattering cross section of the particles, then the general equation for the mean free path $l$ is

$$
l=\frac{0.177}{n \sigma_{s}}=\frac{0.177 k T}{P \sigma_{s}}=2.41 \times 10^{-23} \frac{T}{P \sigma_{s}} \ldots
$$

For fully ionized particles, the cross section $\sigma_{s}$ can be computed approximately according to Lin, Resler, and Kantrowitz (6) as

$$
\sigma_{s}=8.10\left(\frac{e^{2}}{3 k T}\right)^{2} \log \left(\frac{k T}{e \sqrt{4 \pi P}} / \frac{e^{2}}{3 k T}\right) \ldots \ldots
$$

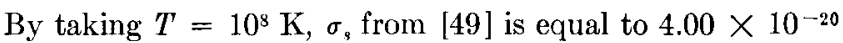
$\mathrm{cm}^{2}$. Then [48] gives a mean free path of $l=603 \mathrm{~cm}$. With such a large free path, the transfer of energy by collisions is extremely slow and inefficient. To improve the chances of collision, some particles of larger size must be introduced, e.g., atoms of heavier elements. The heavier atoms can have their outer electrons stripped (ionized) at the prevailing high temperature, but since some electrons remain attached to the nucleus, the size of the partially ionized atom can still be of the order of $A$. Then such particles will be a scattering cross section of the order of $10^{-16} \mathrm{~cm}^{2}$. Even with only one per cent of such heavier elements in the mixture, the mean free path will be brought down to a few centimeters. This is indeed the mean free path used in the size estimate of the preceding paragraph. Needless to say, the heavier atoms introduced must not capture neutrons appreciably so as not to interfere with the very important energy producing reaction of [41].

However, even with the presence of heavy partially ionized atoms, the mixture will be still practically transparent to high energy neutrons generated by reaction [37]. The energies carried by them cannot then be "kept" in the gas by collision, but rather are received directly by the walls of the reaction chamber. This is a direct energy leak and makes the quenching problem very much more difficult. In fact, out of the reaction [37], only the kinetic energy of ${ }_{2} \mathrm{He}^{3}$ is kept within the gaseous mixture. This energy is only $1 / 4$ of the total given by [40], or $1.31 \times 10^{-6} \mathrm{ergs}$. The energy produced by the reaction [36] is of course retained in the gaseous mixture and is equal to the difference of energies given by [39] and [40] or $1.22 \times 10^{-6}$ ergs. Hence 50 per cent of the deuteron-deuteron reactions have an effective energy production of only

$$
(1.31+1.22) \times 10^{-6}=2.53 \times 10^{-6} \mathrm{ergs} .
$$

The average of the reaction energy kept in the mixture is thus, using [39]

$$
\frac{1}{2}[2.53+6.46] \times 10^{-6} \mathrm{ergs}=4.50 \times 10^{-6} \text { ergs. } .
$$

Compared with gross energy production given by [44], this is only 69.6 per cent; 30.4 per cent of energy produced is delivered directly to the solid walls of the chamber.
Out of the energy kept in the reacting mixture, given by [51] for one single binary reaction or

$$
1.606 \times 10^{10} \mathrm{cal} / \mathrm{gr}
$$

a good fraction will be absorbed by the reacting deuterium in entering the flame: The deuterium gas is heated, dissociated, and finally ionized to reach the full flame temperature of say $1.600 \times 10^{8} \mathrm{~K}$. According to Sänger (1), to heat up to this temperature, the deuterium takes up approximately

$$
10^{9} \mathrm{cal} / \mathrm{gr}=0.1 \times 10^{10} \mathrm{cal} / \mathrm{gr} \ldots \ldots \ldots[53]
$$

Now the crucial question is: how many grams of deuterium have to be heated in order that one gram of deuterium will be burned to completion? In other words, what is the combustion efficiency of the flame in the reaction chamber? By comparing [52] with [53], it is seen that if 16.06 grams of deuterium have to be heated to flame temperature to get one gram of deuterium burned, then there will be no heat left to be conducted and radiated to the wall through the gaseous mixture. But there must be heat conducted and radiated to the wall because the wall, being necessarily of solid material, must be at a temperature, say $2000 \mathrm{~K}$, which is very much lower than the flame temperature of $1.600 \times 10^{8} \mathrm{~K}$. This shows that the ratio of mass to be heated and actually burned must be less than 16.06 .

For lack of more accurate information, consider a combustion efficiency of the flame zone to be $1 / 6$. That is, six grams of deuterium have to be heated to have one gram actually burned. Then the energy available for conduction and radiation to the wall per gram of deuterium burned is, according to [52] and [53]

$$
(1.606-6 \times 0.1) \times 10^{10} \mathrm{cal} / \mathrm{gr}=1.006 \times 10^{10} \mathrm{cal} / \mathrm{gr} . .[54]
$$

Therefore, by comparing with [45], only less than one-half of the gross energy production is available for "cooling" loss. In fact, with [46], the "cooling" loss energy $Q_{c}$ produced per unit vol of flame per unit time is

$$
Q_{c}=\frac{1.006}{2.31} \times 0.874 \mathrm{cal} / \mathrm{cm}^{3} \mathrm{sec}=0.382 \mathrm{cal} / \mathrm{cm}^{3} \mathrm{sec}
$$

Now let it be assumed that the flame in the $100 \mathrm{~m}$ diam reaction chamber be a cylindrical volume of some $60 \mathrm{~m}$ diam and $120 \mathrm{~m}$ long. Then within this $120 \mathrm{~m}$ of flame, the wall will receive by conduction and radiation through the mixture, a heat flux density $q_{c}$ equal to

$$
\begin{aligned}
q_{c}=\frac{\frac{\pi}{4} \times 6000^{2} \times 0.382}{\pi \times 10,000} & =343 \mathrm{cal} / \mathrm{cm}^{2} \mathrm{sec} \\
& =8.75 \mathrm{Btu} / \mathrm{in.} .^{2} \mathrm{sec} .
\end{aligned}
$$

This corresponds to a black body radiation at $3990 \mathrm{~K}$.

The question is, of course, whether the heat flux $q_{c}$ actually equals that given by [56]. For the specified conditions in the reaction chamber, if the actual $q_{c}$ is larger than [56], then the critical reaction chamber diameter must be larger than the assumed $100 \mathrm{~m}$. If less, then the critical diameter can be smaller. Therefore, one of the basic problems of thermonuclear reaction chamber design is the calculation of $q_{c}$ or radiation heat flux through a gas layer of variable composition and variable temperature. The technical complication here is, of course, the fact that here the radiation mean free path is large in comparison with the physical dimensions and therefore the simple method developed by astrophysicists for the interior of stars is not applicable. On the other hand, all essential basic information required for the calculation is now available. The problem is thus only complicated but not insurmountable. But, in any event, the flame is almost transparent due to the low density and almost complete ionization. In fact, within the flame, radiation 
will come almost only from the specially introduced heavy atoms which are, however, of very, very low density. Therefore, the flame, although of extremely high temperature, is a relatively weak radiator. Hence the comparatively low effective black body temperature of $3990 \mathrm{~K}$ may not be far from being correct.

\section{Thermonuclear Power Station}

The part of energy directly transmitted to the wall by fast neutrons is the difference between [45] and [52]; thus the total energy flux to the wall $q$ is

$q=\frac{\frac{\pi}{4} \times 6000^{2} \times\left[0.382+0.874 \times \frac{2.31-1.606}{2.31}\right]}{\pi \times 10,000}=$

$582 \mathrm{cal} / \mathrm{cm}^{2}$ sec $=14.89 \mathrm{Btu} / \mathrm{in}^{2}$ sec $\ldots \ldots[57]$

Although nearly one-half of this energy is realized by slowing down the fast neutrons and is thus distributed in a layer of wall material, not merely delivered at the surface of the wall, nevertheless the tremendous heat density poses a cooling problem which cannot be solved by conventional cooling methods. It seems that the only feasible method is that of transpiration cooling. That is, the wall is made of porous material, say porous carbon or graphite, and cold deuterium gas is forced by pressure through the wall into the reaction chamber. Heat in the wall is picked up by the coolant gas and returned to the reaction chamber. By using a large enough quantity of coolant gas, the wall temperature can be kept at the desired low temperature of, say, $2000 \mathrm{~K}$. In fact, the application of transpiration cooling to nuclear reactors has already been considered by Kaeppeler (7). He, however, has not included the important "spacing heating" effects of neutron slowing-down.

Behind the section of reaction chamber occupied by the flame zone, the heat flux due to neutrons is greatly reduced; then the coolant gas forced into the reaction chamber merely serves to lower the temperature of the gas from the flame zone (exhaust gas). At the end of the reaction chamber, the temperature across the chamber cross section should be fairly uniform and at, say, $1000 \mathrm{~K}$. The discharge pressure of this body of hot gas is of course essentially the chamber pressure which is taken to be $100 \mathrm{~atm}$ in the above discussion. The high pressure hot gas can be used to generate power through a gas turbine. It is perhaps worth while to note that the product gas is expected to contain only the weakly radioactive ${ }_{1} \mathrm{H}^{3}$ and thus should give no difficulty for the power generating machinery. The exhaust from the turbine after being cooled by heat exchanger will pass through the waste extrac-

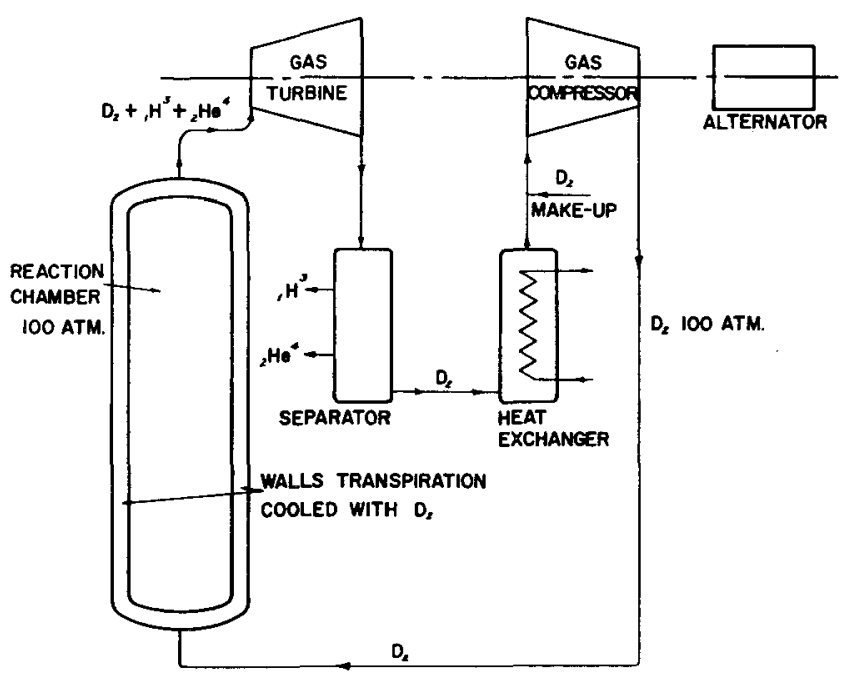

Fig. 1 Schematic diagram of thermonuclear power station tion system for removing the nuclear "ash." The purified gas will contain of course mainly $\mathrm{D}_{2}$, but also has a very small concentration of $\mathrm{H}_{2}$ and $\mathrm{T}_{2}$ produced by reaction [36] and [41]. This gas together with small amount of makeup $\mathrm{D}_{2}$ to replace the deuterium burned is then compressed to high pressure and fed through the porous wall back to the reaction chamber. This then completes the cycle of the power plant. Fig. 1 is a diagram representing the components and the process of the system.

Of course, by cycling the gaseous mixture repeatedly through the reaction chamber, the concentration of $\mathrm{H}_{2}$ and $\mathrm{T}_{2}$ will build up and eventually participate importantly in the energy production through reaction [42], and other reactions. These reactions actually produce the nuclear ash ${ }_{2} \mathrm{He}^{4}$. Finally the composition of feed gas to the reaction chamber will be stabilized at a fixed ratio of $\mathrm{H}_{2}, \mathrm{D}_{2}$, and $\mathrm{T}_{2}$ with the production of $\mathrm{H}$ and $\mathrm{T}$ by reactions [36] and [41] balanced by consumption of $\mathrm{H}$ and $\mathrm{T}$ through reactions [42] and others. Then the over-all result is that of feeding in deuterium $\mathrm{D}_{2}$ and taking out ${ }_{2} \mathrm{He}^{4}$. Thus the reaction chamber effectively converts deuterons to helium according to

$$
{ }_{1} \mathrm{H}^{2}+{ }_{1} \mathrm{H}^{2} \rightarrow{ }_{2} \mathrm{He}^{4}+\text { energy } \ldots
$$

and the energy produced per gram of deuterium burned is very much larger than given by [45]. Furthermore, in this final stage the reaction scheme is considerably more complicated than that discussed in Section 3 and the volume rate of energy generation and heat flux must be somewhat different from that calculated previously. However, these calculations will not be attempted here since the purpose of this study is merely to give a general outline of the problem.

\section{Ignition}

According to the studies made by Sänger (1), the ignition temperature, i.e., the temperature at which the rate of energy production is just balanced by the rate of energy lost, mainly through radiation, with the deuteron-deuteron reaction is approximately $10^{7} \mathrm{~K}$. Naturally the question is how can the thermonuclear reaction be initiated by heating the gaseous mixture to this very high temperature. Before the advent of nuclear fission and the fission bomb, such high temperatures seemed unapproachable. But now this is definitely not so. It may even be possible to obtain ignition without using the fission reaction. But at the moment, one can only say that ignition of the thermonuclear reaction is certainly possible; no detailed scheme can, however, be suggested.

\section{Thermonuclear Power Industry}

The rate of energy production $Q$ according to [46] is 0.00365 $\mathrm{kw} / \mathrm{cm}^{3}$ in the flame zone. If, as assumed previously, the flame zone is a cylinder of $60 \mathrm{~m}$ diam and $120 \mathrm{~m}$ long, the total energy production is

$$
0.00365 \times(\pi / 4) \times 6000^{2} \times 12,000=1.238 \times 10^{9} \mathrm{kw} \text {. [59] }
$$

If the thermodynamic efficiency of the power plant cycle is 25 per cent, the power of the station is

$$
0.25 \times 1.238 \times 10^{9} \mathrm{kw}=0.309 \times 10^{9} \mathrm{kw} \ldots \ldots[60]
$$

Thus continuous operation of the plant will product annually electric energy of the amount

$$
0.309 \times 10^{9} \times 24 \times 365=2.71 \times 10^{12} \mathrm{kw} \mathrm{hr} \ldots[61]
$$

In 1954, the annual electric energy production in the United States was approximately $0.5 \times 10^{12} \mathrm{kw} \mathrm{hr}$. Thus in one thermonuclear power plant, perhaps one of minimum size, the capacity is over five times the total effective capacity of the United States! This points to the extreme importance of determining the critical quenching size of the thermonuclear reaction chamber accurately. The speculations in the pre- 
ceding sections are based upon an assumed diameter of $100 \mathrm{~m}$ for the reaction chamber. Smaller size and lower flame temperature will naturally reduce the scale of the power plant. However for the deuteron-deuteron reaction, the ignition temperature is roughly $10^{7} \mathrm{~K}$. For steady burning, the flame temperature cannot be below this temperature.

To conclude this discussion, comparison of thermonuclear energy and other energy sources will be made: According to [45], the fusion energy of deuterium is

$2.31 \times 10^{10} \times 1.8 \mathrm{Btu} / \mathrm{lb}=4.16 \times 10^{10} \mathrm{Btu} / \mathrm{lb}$ of $\mathrm{D}_{2} \ldots[62]$

The fission of one pound of U-235 gives $3.14 \times 10^{10} \mathrm{Btu}$. Therefore fusion energy is almost $4 / 3$ times as large as fission energy. Since the natural isotope concentration of deuterium in hydrogen is $1: 7000$, in terms of natural hydrogen, the fusion energy is

$\left(4.16 \times 10^{10}\right) / 7000=5.94 \times 10^{6} \mathrm{Btu} / \mathrm{lb}$ of hydrogen. .63$]$ or, referred to water

$$
(5.94 \times 10) / 9=6.60 \times 10^{5} \mathrm{Btu} / \mathrm{lb} \text { of } \mathrm{H}_{2} \mathrm{O} \ldots \ldots[64]
$$

If the average chemical energy of coal is taken as $11,000 \mathrm{Btu} /$ $\mathrm{lb}$, one pound of water is potentially equivalent to sixty pounds of coal! But even all this is based upon only partial burning of deuteron to triton and proton. With complete burning into ${ }_{2} \mathrm{He}^{4}$, the thermonuclear energy of deuterium will be still larger. Therefore, if thermonuclear power plants can actually be constructed, then the source of fusion energy far exceeds the other terrestial energy resources, chemical or fission.

\section{References}

1 Sänger, E., Astronautica Acta, vol. 1, 1955, pp. 61-88.

2 Kaeppeler, H. J., J. Astronautics, vol. 2, 1955, pp. 50-56.
3 Gamow, G., and Teller, E., Physical Review, vol. 53, 1938, p. 608. Cf. Gamow, G., Critchfield, C. L., "Theory of Atomic Nucleus and Nuclear Energy Sources," Oxford, 1949, chap. 10. 4 Fowler, R. H., and Guggenheim, E. A., "Statistical Thermodynamies," Cambridge, 1939, p. 493.

5 Arnold, W. R., Phillips, J. A., Sawyer, G. A., Stovall, E. J., Jr., and Tuck, J. L., Physical Review, vol. 93, 1954, p. 483.

6 Lin, S.-C., Resler, E. L., and Kantrowitz, A., Journal of A pplied Physics, vol. 26, 1955, p. 95.

7 Kaeppeler, H. J., Jet Propulsion, vol. 24, 1954, p. 316.

\section{Comments on Hsue-shen Tsien Paper ${ }^{1}$}

\section{JESSE L. GREENSTEIN ${ }^{2}$}

Mount Wilson and Palomar Observatories, Pasadena, Calif.

D R. TSIEN had little contact with physicists or astronomers during his studies of a thermonuclear power plant, so that the methods used in his derivation of reaction yields are not the most modern. The concept of effective collision diameter $[9,11]$ is far from precise; however, as pointed out following [22] it is necessary to fit the experimental data empirically, so that essentially only the forms of $[16,22]$ are needed. This fitting, or even a different integration method,

(Continued on page 575)

${ }^{1}$ Prof. Greenstein had been asked by the editors to review the Tsien paper. His report recommended that it be published, but he expressed reservations about some aspects of the analysis. Because of Dr. Tsien's absence from the country, the points at issue could not be resolved in the usual manner by private correspondence. Accordingly, Prof. Greenstein gave us his permission to publish his comments, and it is left to the reader to form his own conclusions.

${ }^{2}$ Professor, California Institute of Technology. 